\title{
High-refractive index of acrylate embedding resin clarifies mouse brain tissue
}

Hongfu Zhou

Yumiao Xiong

Yu Wang

Xiaojun Wang

Pei Li

Yadong Gang

Xiuli Liu

Shaoqun Zeng 


\section{High-refractive index of acrylate embedding resin clarifies mouse brain tissue}

\author{
Hongfu Zhou, a,b,t Yumiao Xiong, ${ }^{\text {a,b, }, \dagger}$ Yu Wang, ${ }^{\text {a,b }}$ \\ Xiaojun Wang, ${ }^{\text {a,b }}$ Pei Li, ${ }^{a, b}$ Yadong Gang, ${ }^{\text {a,b }}$ \\ Xiuli Liu, ${ }^{\mathrm{a}, \mathrm{b}}$ and Shaoqun Zeng ${ }^{\mathrm{a}, \mathrm{b}, *}$ \\ aWuhan National Laboratory for Optoelectronics-Huazhong \\ University of Science and Technology, Britton Chance Center for \\ Biomedical Photonics, Wuhan, China \\ bHuazhong University of Science and Technology, \\ Ministry of Education Key Laboratory for Biomedical Photonics, \\ Collaborative Innovation Center for Biomedical Engineering, \\ School of Engineering Sciences, Wuhan, China
}

\begin{abstract}
Biological tissue transparency combined with light-sheet fluorescence microscopy is a useful method for studying the neural structure of biological tissues. The development of light-sheet fluorescence microscopy also promotes progress in biological tissue clearing methods. The current clarifying methods mostly use liquid reagent to denature protein or remove lipids first, to eliminate or reduce the scattering index or refractive index of the biological tissue. However, denaturing protein and removing lipids require complex procedures or an extended time period. Therefore, here we have developed acrylate resin with a high refractive index, which causes clearing of biological tissue directly after polymerization. This method can improve endogenous fluorescence retention by adjusting the $\mathrm{pH}$ value of the resin monomer. $\odot$ The Authors. Published by SPIE under a Creative Commons Attribution 3.0 Unported License. Distribution or reproduction of this work in whole or in part requires full attribution of the original publication, including its DOI. [DOI: 10.1117/1.JBO.22.11.110503]
\end{abstract}

Keywords: embedding resin; endogenous fluorescence; refractive index; tissue clearing.

Paper 170438LRR received Jul. 5, 2017; accepted for publication Oct. 6, 2017; published online Nov. 16, 2017.

Biological tissue transparency combined with light-sheet fluorescent microscopy has become an important method for studying the neural structure of biological tissue, and methods for clarifying biological tissue have advanced greatly. ${ }^{1-3}$ Biological tissue transparency is mainly attained via denaturation of protein or removal of lipids of the cell membrane, to eliminate or reduce the refractive index or the scattering index of the biological tissue, so that the deeper organizational structure of nerves can be observed. ${ }^{4,5}$ The recently developed clarifying methods can be divided into three categories: aqueous-based clearing, hydrogel embedding combined with electrophoresis clearing, and solvent-based clearing. Aqueous-based

*Address all correspondence to: Shaoqun Zeng, E-mail: sqzeng@mail.hust. edu.cn

†These authors contributed equally to this work. clearing of biological tissues tends to cause tissue expansion and requires an extended period of time, and some denaturing reagents, such as urea or sodium dodecyl sulfate, could cause the tissue to become antigen-insensitive. ${ }^{6-13}$ Hydrogel embedding combined with electrophoresis clearing is a complex method that requires gel embedding and electrophoresis equipment, and the reproducibility of experimental results is poor. ${ }^{14-16}$ Solvent-based reagents virtually quench endogenous fluorescent proteins. ${ }^{17-20}$ Although the ultimate three-dimensional imaging of solvent cleared organs technique can clarify biological tissues expressing fluorescent proteins, the fluorescence intensity gradually diminishes after clearing. ${ }^{21}$ To resolve these problems, the fluorescent signal requires long-term preservation and antibleaching treatment after resin embedding; this method involves first using solvent-based clearing of the biological tissue, after which it is embedded in epoxy resin. ${ }^{22}$

We developed an acrylic resin with a high refractive index resin (termed HRAR) that clears biological tissue directly after embedding. The refractive index is increased when the liquid monomer polymerizes into solid resin, which matches the refractive index of the dehydrated biological tissue perfectly, to achieve transparency. This method can clear large samples directly without requiring special pretreatment, and can maintain the endogenous fluorescent signal well. In this study, we investigated the effect of differences in the $\mathrm{pH}$ value of monomers on green fluorescent protein (GFP) fluorescence, ${ }^{23}$ and a transparent GFP mouse brain block imaged via light-sheet fluorescence microscopy.

Monomer 2-phenoxyethyl acrylate and the crosslinking agent ethylene glycol dimethacrylate (mass ratio of 9:1) were polymerized in the presence of $0.2 \%$ initiator $2,2^{\prime}$-azobis(2,4-dimethyl) valeronitrile to produce HRAR resin (Fig. 1). The main effect of 2-phenoxyethyl acrylate is to increase the refractive index after polymerization, and the crosslinking agent triethylene glycol dimethacrylate causes the resin to form a crosslinked network structure that improves the hardness of the resin. The results (Fig. 2) showed that the light transmittance of an HM20 resin-embedded mouse brain was about $7 \%$ in the spectral range of 400 to $1100 \mathrm{~nm}$, while the transmittance of an HRAR resin-embedded mouse brain was about $70 \%$ in this spectral range.

Eight-week-old mice were perfused according to the recommended protocol for the perfusion of mice. ${ }^{24}$ Animal care and use were in accordance with the guidelines of the Administration Committee of Affairs Concerning Experimental Animals in Hubei Province of China. The protocol was approved by the Committee on the Ethics of Animal Experiments of the Huazhong University of Science and Technology (permit number: 00027340). All efforts were made to minimize the suffering of the animals. The tissue was sectioned into $100-\mu \mathrm{m}$ coronal slices using a vibrating microtome (Leica, VT1000 S), and the slices were stored in $0.01 \mathrm{M}$ PBS solution at $4^{\circ} \mathrm{C}$ in the dark.

The $100-\mu \mathrm{m}$ brain slices were dehydrated according to the following procedure: $75 \%$ tetrahydrofuran (THF) for $3 \mathrm{~min}$, $100 \%$ THF for $3 \mathrm{~min}$, and $100 \% \mathrm{THF}$ for $4 \mathrm{~min}$. The brain slices were then placed in the resin mixture twice for 5 min each time. Two drops of resin mixture were then added to the slides, which were subsequently coverslipped. The brain slices on the slides were polymerized in an oven at $45^{\circ} \mathrm{C}$ for $8 \mathrm{~h}$. This experiment was carried out to verify the endogenous fluorescence intensity before and after embedding in the HRAR resin. First, $100-\mu \mathrm{m}$ brain slices were placed on a slide and coverslipped. Confocal 


\section{JBO Letters}

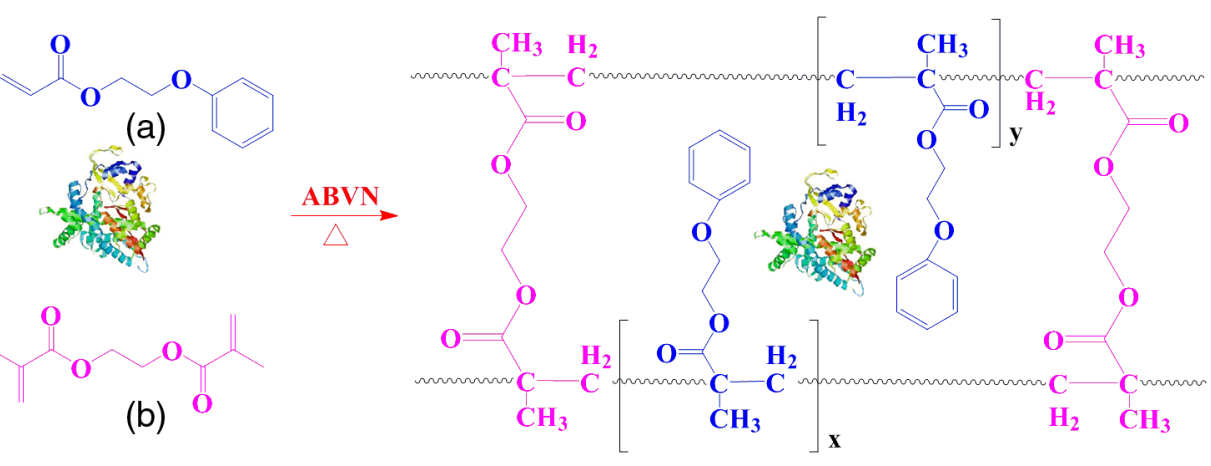

Fig. 1 The polymerization of HRAR. The structure (a) is monomer 2-phenoxyethyl acrylate, the structure (b) is crosslinking agent ethylene glycol dimethacrylate, and the protein structure represents the embedded biological tissue.
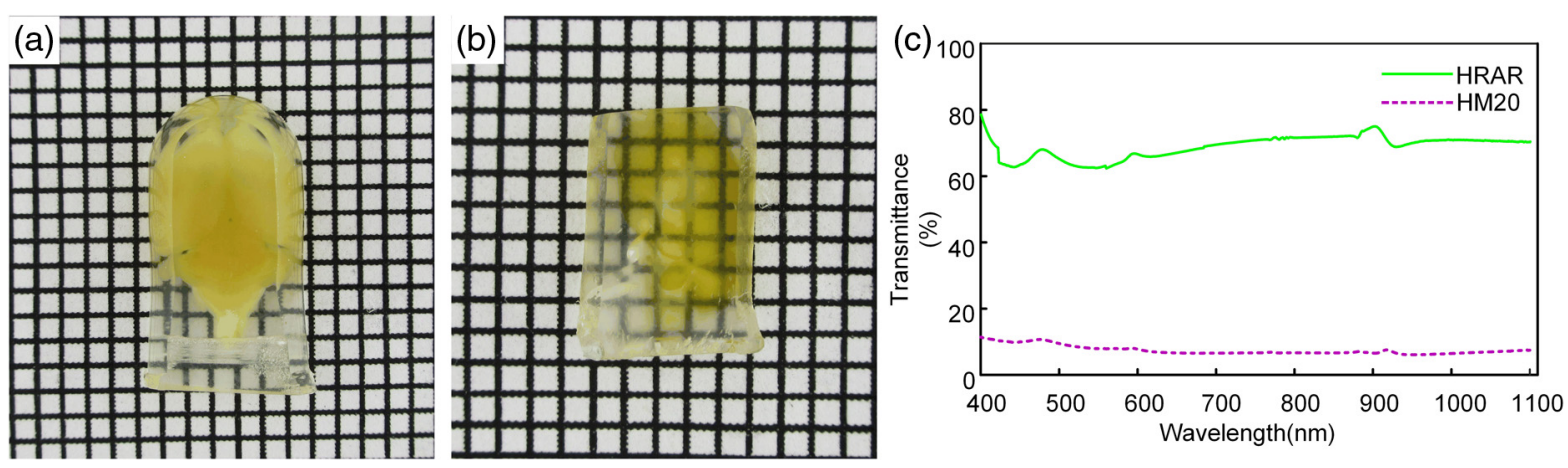

Fig. 2 The clearing effect of HRAR. (a) HM 20 resin-embedded mouse brain block; (b) HRAR resinembedded mouse brain block; (c) optical transmittance of HM20 resin- and HRAR resin-embedded mouse brain blocks. A PerkinElmer (Waltham, Massachusetts) Lambda $35 \mathrm{UV} / \mathrm{vis}$ spectrometer was used at an excitation bandwidth of $0.5 \mathrm{~nm}$, with a spectral range of 400 to $1100 \mathrm{~nm}$ was used to assess transmittance of an HM2O resin block and an HRAR resin block with 3-mm thickness.

fluorescence microscopy (Zeiss 780) was then used to image the region of interest. The imaged slices were embedded according to the above procedure, and confocal fluorescence microscopy (Zeiss 780) was again used to image the same region using the same parameters. To quantitatively evaluate the endogenous fluorescence intensity of the tissue before and after embedding in the HRAR resin, we used the fluorescence intensity of the soma as an indicator. First, the imaging data were imported into Image $\mathbf{J}$ software. Within this software, the rectangularselection tool was activated, a circular area on the soma was selected, and the histogram tool was then used to measure the average gray value; the average gray value of the soma before embedding was denoted as $A$, while the average gray value of the soma after embedding was denoted as $B$. The fluorescence retention rate after embedding was calculated as follows: $B / A \times 100 \%$. For each group of experiments, we selected 10 somata to evaluate the average gray values.

2-phenoxyethyl acrylate and ethylene glycol dimethacrylate were filtered through alkaline alumina $\left(\mathrm{Al}_{2} \mathrm{O}_{3}\right)$, respectively, solution a was prepared by mixing 2-phenoxyethyl acrylate and ethylene glycol dimethacrylate in a mass ratio of $9: 1$, then $0.2 \%$ initiator $2,2^{\prime}$-azobis(2,4-dimethyl) valeronitrile was added, at last the mixture was stirred for $30 \mathrm{~min}$; solution $b$ was prepared by mixing 2-phenoxyethyl acrylate and ethylene glycol dimethacrylate in a mass ratio of $9: 1,10 \%$ tetrasodium ethylenediaminetetraacetate dehydrate $\left(\right.$ EDTA- $\left.\mathrm{Na}_{4}\right)$ was then added and the solution was shaken for $6 \mathrm{~h}$. The mixture was filtered and then $0.2 \%$ initiator $2,2^{\prime}$-azobis(2,4-dimethyl) valeronitrile was added, at last the mixture was stirred for 30 min. Solution c was prepared by mixing 2-phenoxyethyl acrylate and ethylene glycol dimethacrylate in a mass ratio of $9: 1,10 \%$ tetrasodium ethylenediaminetetraacetate dehydrate (EDTA- $\mathrm{Na}_{4}$ ) was then added and the solution was shaken for $6 \mathrm{~h}$. The mixture was filtered and then $0.2 \%$ initiator $2,2^{\prime}$-azobis(2,4-dimethyl) valeronitrile and $0.5 \%$ o 2,2'-diaminodiethylamine were added, at last the mixture was stirred for $30 \mathrm{~min}$. The three solutions that were measured with a $\mathrm{pH}$ meter (OHAUS S-5000 pH meter, Parsippany, New Jersey) were calculated the average $\mathrm{pH}$ value after multiple measurements. The results (Fig. 3) show that the fluorescence retention ratio of endogenous GFP signal was $46 \%$ when the $\mathrm{pH}$ value of the resin monomer was $4.81,162 \%$ when the $\mathrm{pH}$ value of the resin monomer was 6.27 , and $210 \%$ when the $\mathrm{pH}$ value of the resin monomer was 9.62. This indicates that the $\mathrm{pH}$ value of the resin monomer affected the fluorescence signal intensity markedly, and that an alkaline environment is beneficial to the maintenance of the endogenous GFP signal.

The postfixed mouse brain block was rinsed three times with $0.01 \mathrm{M}$ PBS solution for $8 \mathrm{~h}$ each at $4^{\circ} \mathrm{C}$ in the dark, after which it was dehydrated in 50\% THF for $2 \mathrm{~h}, 75 \%$ THF for $2 \mathrm{~h}, 95 \%$ THF for $2 \mathrm{~h}, 100 \%$ THF for $3 \mathrm{~h}$, and $100 \%$ THF for $3 \mathrm{~h}$ at $4^{\circ} \mathrm{C}$. The dehydrated brain block was then embedded in HRAR resin as follows: HRAR resin for $2 \mathrm{~h}$, HRAR resin for $24 \mathrm{~h}$, and HRAR resin for $48 \mathrm{~h}$ at $4^{\circ} \mathrm{C}$ in the dark. Next, the permeated 

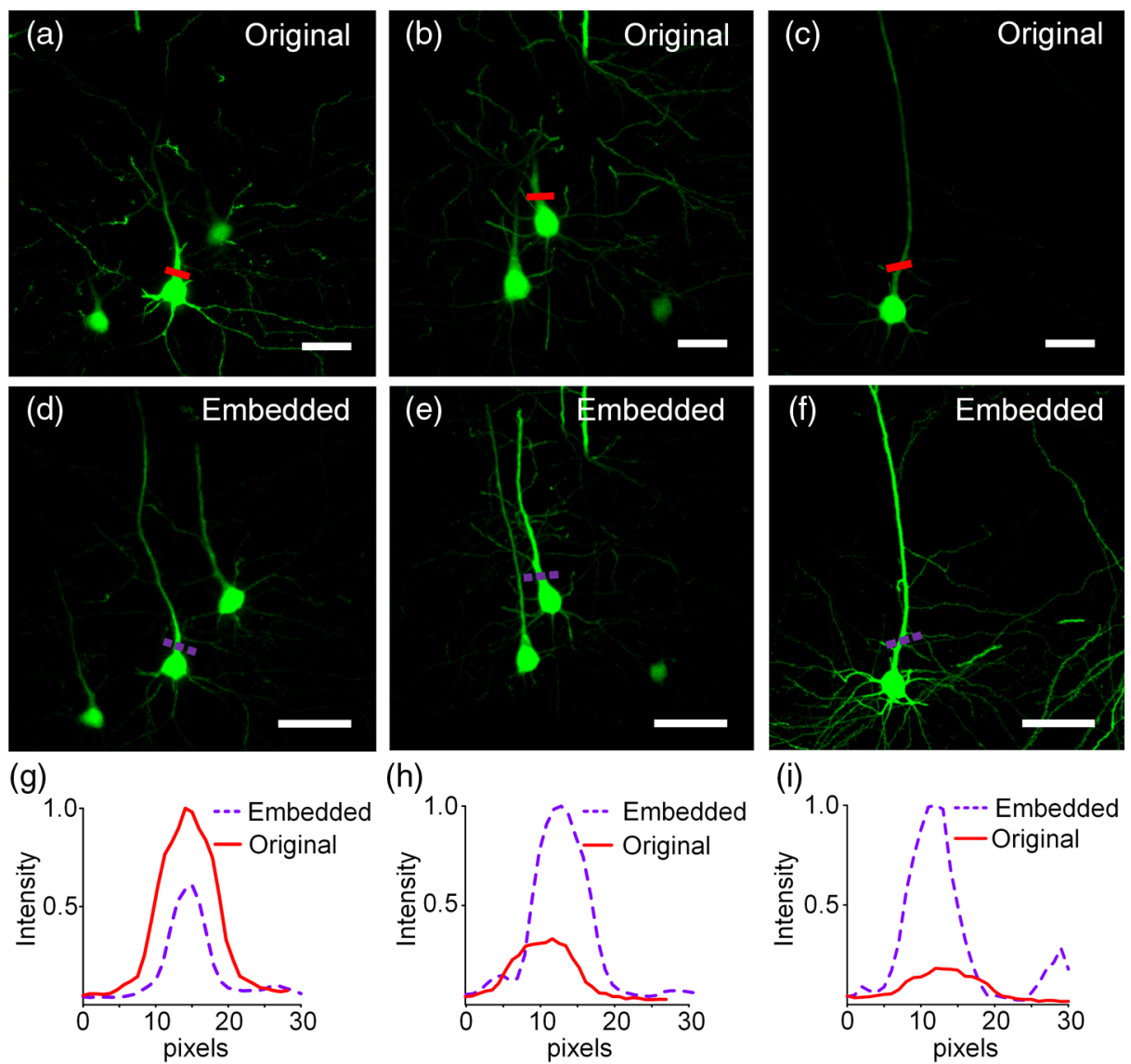

(h)
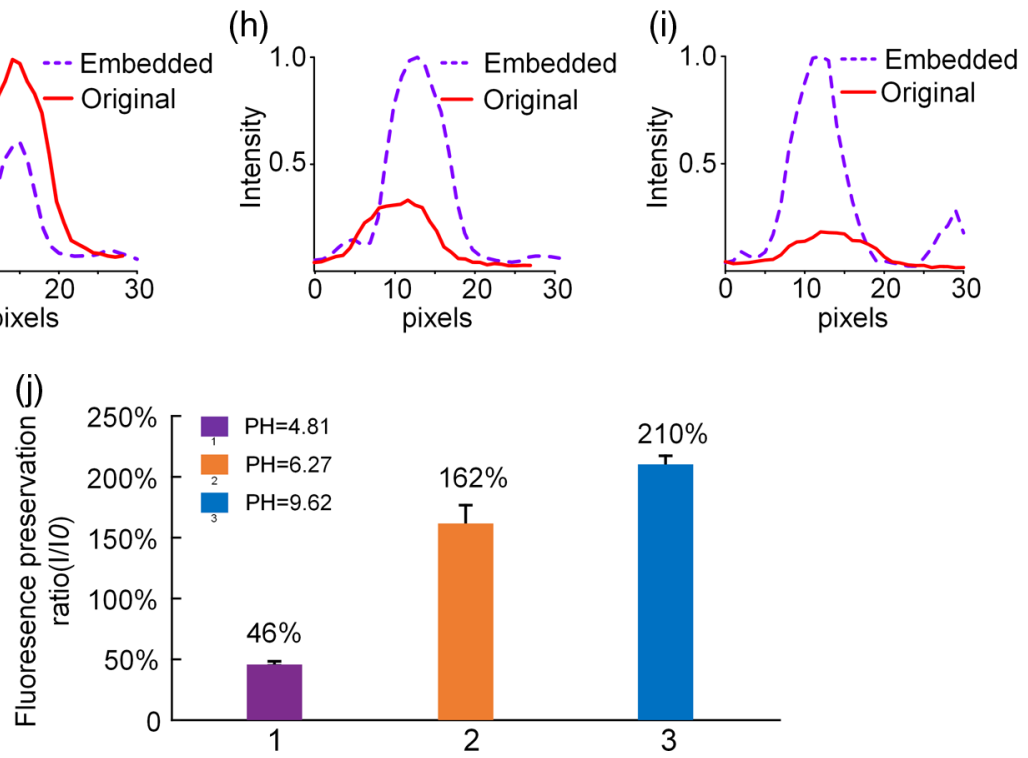

Fig. 3 The fluorescence comparison of GFP in brain slices after embedding in resin with different $\mathrm{pH}$ values. (a), (b), and (c) The imaging results of the brain slices in PBS buffer. (d) The imaging results after embedding the tissue in HRAR liquid agent at a $\mathrm{pH}$ of 4.81. (g) The fluorescence intensity distribution curve for the area indicated by colored lines in (a) and (d). (e) The imaging results after embedding tissue in HRAR liquid agent at a pH of 6.27. (h) The fluorescence intensity distribution curve for the area indicated by colored lines in (b) and (e). (f) The imaging results after embedding in HRAR liquid agent at a pH of 9.62. (i) The fluorescence intensity distribution curve for the area indicated by colored lines in (c) and (f). (j) The fluorescence intensity ratio of GFP before and after embedding of tissue in HRAR resin; the pH of column 1 is 4.81 , the $\mathrm{pH}$ of column 2 is 6.27 , and the $\mathrm{pH}$ of column 3 is 9.62 , and the scale bar for all images (a)-(f) indicates $30 \mu \mathrm{m}$. As the tissue block shrink around $20 \%$ in each direction after embedding, (d)-(f) enlarged accordingly to make the soma be close to that of the original image. Therefore, the scale bar of (d)-(f) is longer than that in (a)-(c). For (a)-(f), three-dimensional images were obtained with tissue thickness of $100 \mu \mathrm{m}$, at a $z$-step of $1 \mu \mathrm{m}$, using a confocal fluorescence microscopy (Zeiss 780), equipped with a 488-nm laser, a $20 \times$ objective lens with numerical aperture of 1.0, zoom of 1.0, medium is water. (a)-(f) $z$-axis maximum projection images of these images stacks.

mouse brain block was placed in a capsule that was filled with HRAR resin. The curing procedure was as follows: $32^{\circ} \mathrm{C}$ for $8 \mathrm{~h}$, $38^{\circ} \mathrm{C}$ for $8 \mathrm{~h}, 45^{\circ} \mathrm{C}$ for $8 \mathrm{~h}$, and $50^{\circ} \mathrm{C}$ for $4 \mathrm{~h}$ in vacuum and dark conditions. The results (Fig. 4) from light-sheet fluorescence microscopy imaging showed that the fluorescence signal and neuronal fiber structure were well preserved with the HRAR resin (solution c), and that neuronal fibers were not distorted by this embedding process. Thus, the polymerization rate was uniform, and this method could be applied for clearing large samples.

In this paper, we applied the principle of refractive index matching to clear biological tissue, and found that the refractive 

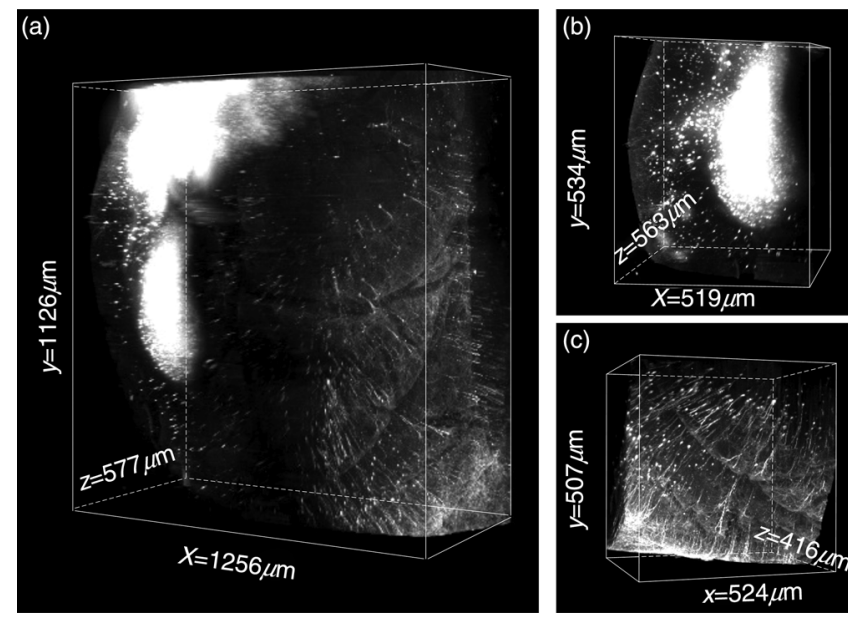

Fig. 4 (a) Light-sheet fluorescence microscopy imaging of an HRAR resin-embedded GFP brain block. (b) and (c) Magnified cortex nerve three-dimensional structure. Light-sheet fluorescence microscope (Ultramicroscope, LaVision BioTec, Bielefeld, Germany), equipped with a $488-n m$ laser, $2 \times$ objective lens with numerical aperture of 0.5 , zoom of 1.6 , in dibenzyl ether medium.

index increased by about 0.05 after the acrylic monomer polymerized. We then developed an acrylate resin with a high refractive index that could clear dehydrated biological tissue directly. The average refractive index of the resin monomer is 1.512 , and the biological tissue was translucent after penetration by the resin monomer. The average refractive index of the polymerized resin is 1.558 , and the refractive index of the dehydrated biological tissue is 1.55 to $1.56,{ }^{1}$ the refractive index of the acrylate resin, therefore, matches that of the dehydrated biological tissue, which results in direct clearing of the biological tissue after polymerization. The refractive index of a traditional commercial acrylic monomer is about 1.40 to 1.45 , and the refractive index of the solid commercial resin is about 1.50. Therefore, biological tissue embedded in such resin cannot be transparent, as the refractive index of the commercial resin is mismatched to that of the dehydrated biological tissue.

In contrast with traditional commercial acrylate resin, the HRAR resin greatly improved the ability of light to penetrate biological tissue. The light transmittance in the spectral range of 400 to $1100 \mathrm{~nm}$ does not change markedly, which ensured that standard light wavelengths could penetrate the embedded biological tissue, greatly reducing attenuation of the excitation and emission light.

We also investigated the fluorescence retention by adjusting the $\mathrm{pH}$ value of the resin monomer, and found that an alkaline environment is beneficial to maintaining endogenous GFP signals, while an acidic environment could quench endogenous GFP signals. ${ }^{23}$ Thus, the $\mathrm{pH}$ value of the resin monomer can affect endogenous GFP signal intensity markedly. In our study, solution $\mathrm{c}$ was the best for maintaining the GFP signal. Lightsheet microscopy imaging of brain block showed that the cell bodies and nerve fibers were maintained intact, indicating that this method is also suitable for large sample clearing and light-sheet fluorescent microscopy imaging.

\section{Disclosures}

The authors have no relevant financial interests in this article and no potential conflicts of interest to disclose.

\section{Acknowledgments}

We thank Jianyi Xu, Fang Fang Yin, Yadong Gang, Pei Li, Ke Bai, Lin Li, and Yuanlei Yue for their helpful comments. This work was supported by the NSFC Project Nos. 81327802 and 61421064 .

\section{References}

1. D. S. Richardson and J. W. Lichtman, "Clarifying tissue clearing," Cell 162(2), 246-257 (2015).

2. A. Feuchtinger, A. Walch, and M. Dobosz, "Deep tissue imaging: a review from a preclinical cancer research perspective," Histochem. Cell Biol. 146(6), 781-806 (2016).

3. B.-C. Chen et al., "Lattice light-sheet microscopy: imaging molecules to embryos at high spatiotemporal resolution," Science 346(6208), 1257998 (2015)

4. H. Liu et al., "Dependence of tissue optical properties on solute-induced changes in refractive index and osmolarity," J. Biomed. Opt. 1(2), 200 211 (1996).

5. S. L. Jacques, "Corrigendum: optical properties of biological tissues: a review," Phys. Med. Biol. 58(14), 5007-5008 (2013).

6. Y.-C. Liu and A.-S. Chiang, "High-resolution confocal imaging and three-dimensional rendering," Methods 30(1), 86-93 (2003).

7. P. S. Tsai et al., "Correlations of neuronal and microvascular densities in murine cortex revealed by direct counting and colocalization of nuclei and vessels," J. Neuroscience 29(46), 14553-14570 (2009).

8. H. Hama et al., "Scale: a chemical approach for fluorescence imaging and reconstruction of transparent mouse brain," Nat. Neurosci. 14(11), 1481-1488 (2011)

9. T. Kuwajima et al., "Clear" ${ }^{\mathrm{T}}$ a detergent and solvent-free clearing method for neuronal and non-neuronal tissue," Development 140(6), 1364-1368 (2013)

10. K. Tainaka et al., "Whole-body imaging with single-cell resolution by tissue decolorization," Cell 159(4), 911-924 (2014).

11. B. Yang et al., "Single-cell phenotyping within transparent intact tissue through whole-body clearing," Cell 158(4), 945-958 (2014).

12. B. Hou et al., "Scalable and DiI-compatible optical clearance of the mammalian brain," Front. Neuroanat. 9, 1-19 (2015).

13. Y. Aoyagi et al., "A rapid optical clearing protocol using $2,2^{\prime}$-thiodiethanol for microscopic observation of fixed mouse brain," PLoS One 10(1), e0116280 (2015).

14. K. Chung et al., "Structural and molecular interrogation of intact biological systems," Nature 497(7449), 332-337 (2013).

15. S.-Y. Kima et al., "Stochastic electrotransport selectively enhances the transport of highly electromobile molecules," Proc. Natl. Acad. Sci. U.S.A. 112(46), E6274-E6283 (2015).

16. E. Lee et al., "ACT-PRESTO: rapid and consistent tissue clearing and labeling method for 3-dimensional (3D) imaging," Sci. Rep. 6, 18631 (2016).

17. W. Spalteholz, Uber das Durchsichtigmachen von menschlichen und tierischen Praparaten, S. Hierzel, Leipzig (1914).

18. H.-U. Dodt et al., "Ultramicroscopy: three-dimensional visualization of neuronal networks in the whole mouse brain," Nat. Methods 4(4), 331-336 (2007).

19. A. Ertürk et al., "Three-dimensional imaging of solvent-cleared organs using 3DISCO," Nat. Protoc. 7(11), 1983-1995 (2012).

20. N. Renier et al., "iDISCO: a simple, rapid method to immunolabel large tissue samples for volume imaging," Cell 159(4), 896-910 (2014).

21. C. Pan et al., "Shrinkage-mediated imaging of entire organs and organisms using uDISCO," Nat. Methods 13(10), 859-867 (2016).

22. K. Becker et al., "Reduction of photo bleaching and long term archiving of chemically cleared GFP-expressing mouse brains," PLoS One 9(12), e114149 (2014)

23. Z. Yang et al., "Development of a plastic embedding method for large volume and fluorescent-protein-expressing tissues," PLoS One 8(4), e60877 (2013).

24. Y. Gang et al., "Embedding and chemical reactivation of green fluorescent protein in the whole mouse brain for optical micro-imaging," Front. Neurosci. 11, 121 (2017). 